Meta

Journal des traducteurs

Translators' Journal

\title{
Approche argumentative des textes scientifiques : la traduction de or en espagnol
}

\section{Joëlle Rey}

Volume 44, numéro 3, septembre 1999

URI : https://id.erudit.org/iderudit/003946ar

DOI : https://doi.org/10.7202/003946ar

Aller au sommaire du numéro

\section{Éditeur(s)}

Les Presses de l'Université de Montréal

ISSN

0026-0452 (imprimé)

1492-1421 (numérique)

Découvrir la revue

Citer cet article

Rey, J. (1999). Approche argumentative des textes scientifiques : la traduction de or en espagnol. Meta, 44(3), 411-428. https://doi.org/10.7202/003946ar
Résumé de l'article

À partir de l'étude d'un corpus de textes scientifiques français et de leur traduction en espagnol, le connecteur français OR et les équivalents espagnols qu'il peut recevoir sont analysés dans une perspective argumentative. Après une brève présentation de l'étymologie et des valeurs de base de $\mathrm{OR}$, son fonctionnement en contexte est examiné, ce qui permet de dégager les valeurs contextuelles de ce connecteur et de les catégoriser. Les critères ayant servi de base à cette catégorisation sont alors utilisés pour évaluer le choix du connecteur proposé dans la traduction espagnole et, le cas échéant, suggérer d'autres solutions. 


\title{
Approche argumentative des textes scientifiques: la traduction de or en espagnol'
}

\author{
joëlle rey \\ Université Pompeu Fabra, \\ Barcelone, Espagne
}

\begin{abstract}
RÉSUMÉ
À partir de l'étude d'un corpus de textes scientifiques français et de leur traduction en espagnol, le connecteur français $O R$ et les équivalents espagnols qu'il peut recevoir sont analysés dans une perspective argumentative. Après une brève présentation de l'étymologie et des valeurs de base de $O R$, son fonctionnement en contexte est examiné, ce qui permet de dégager les valeurs contextuelles de ce connecteur et de les catégoriser. Les critères ayant servi de base à cette catégorisation sont alors utilisés pour évaluer le choix du connecteur proposé dans la traduction espagnole et, le cas échéant, suggérer d'autres solutions.
\end{abstract}

\begin{abstract}
This paper presents an argumentation-based analysis of the French connective $O R$ with Spanish equivalents, taken from a corpus of French scientific texts with corresponding Spanish translations. Following a brief discussion of the connective's etymology and semantic values, an analysis is made of its contextual function. This analysis provides identification of the contextual values and categorises them. The resulting categorisation criteria are used as the basis for an assessment of the choice of connectives in the corresponding Spanish translations, as well as suggesting possible alternatives.
\end{abstract}

\section{INTRODUCTION}

Dans cet article, $j$ 'analyse la traduction du connecteur $O R$ dans une perspective pragmatique, et plus précisément à partir des notions dégagées par O. Ducrot et J. C. Anscombre dans leur "Théorie de l'argumentation et de la polyphonie». Sans entrer dans les détails de cette théorie, je rappelle simplement qu'elle est fondée sur le postulat que tout énoncé est orienté vers une certaine classe de conclusions et est, par conséquent, essentiellement argumentatif. Ceci amène Ducrot (1982: 143) à définir l'argumentation en ces termes:

Je parlerai d'argumentation chaque fois qu'un même locuteur prend en charge deux ensembles, A et C, d'énoncés, en présentant l'un comme destiné à faire admettre l'autre (il est possible que chacun des ensembles se réduise à un énoncé unique, comme dans «Il fait mauvais: je reste à la maison»). J'appelle «conclusions» les énoncés — par convention je les représenterai par la lettre $" \mathrm{C} »-$ que l'on dit vouloir faire admettre, et «arguments» - par convention « $\mathrm{A}$ » — ceux qui sont censés autoriser les autres.

De cette définition surgissent alors les concepts de visée argumentative et d'orientation argumentative. Selon Ducrot,

Dire maintenant qu'un énoncé comporte une ou plusieurs visées argumentatives, c'est dire qu'il présente certains des éléments sémantiques qu'il contient comme susceptibles, 
[...], de faire admettre, en suivant un trajet déterminé, telles ou telles conclusions.

(Ducrot, 1983: 7)

Ce trajet argumentatif devient plus complexe lorsqu'il y a plusieurs ensembles d'énoncés et une seule conclusion. Étant donné un premier ensemble d'énoncés A et un ensemble de conclusions $C$, tout ensemble d'énoncés B venant compléter A sera aussi orienté vers une classe de conclusions. Deux possibilités se présentent alors: soit la classe d'énoncés B tend aussi vers la classe de conclusions $\mathrm{C}$ et on parlera de coorientation, soit la classe d'énoncés B tend vers une classe de conclusions non-C et on parlera d'anti-orientation.

Le problème qui se pose alors est d'identifier les unités linguistiques porteuses de ces orientations argumentatives. Selon Ducrot, l'orientation est inscrite à la fois dans l'énoncé lui-même et dans des marques linguistiques spécifiques: les connecteurs, qui articulent les différents énoncés.

Le connecteur $O R$ est relativement peu fréquent en français écrit, rare en dialogue, mais très fréquent dans les textes de type scientifique qui ont servi de base à cette étude. Il s'agit en effet d'un terme courant en logique et qui fait intégralement partie de la phraséologie des livres de mathématiques et autres domaines reposant sur le raisonnement. De plus, c'est un terme qui, en fonction du contexte, peut recevoir des équivalents divers en espagnol, ce qui le rend particulièrement intéressant du point de vue de la traduction.

\section{1. ÉTYMOLOGIE ET DÉFINITIONS DU CONNECTEUR OR}

Le morphème $O R$ vient de l'expression latine hac hora, «à cette heure ». Il surgit tout d'abord sous la forme $a$ ore; puis, comme contraction de cette dernière, on le trouve dès la fin $\mathrm{du} \mathrm{x}^{\mathrm{e}}$ siècle sous la forme hor, or; vers le $\mathrm{xi}^{\mathrm{e}}$ siècle, il devient ore, et au $\mathrm{xi}^{\mathrm{e}}$ siècle, ores. Jusqu'à la fin du $\mathrm{xi}^{\mathrm{e}}$ siècle, il a seulement la fonction d'adverbe temporel avec le sens de «maintenant».

À partir du xii ${ }^{\mathrm{e}}$ siècle, il acquiert une nouvelle valeur qu'il conservera jusqu'à nos jours : il commence à être utilisé comme conjonction servant à «amener un élément nouveau dans un récit ou dans la chaîne d'un raisonnement, en opposition plus ou moins nette avec les éléments précédents », comme le définit le Grand dictionnaire Larousse de la langue française. Enfin, toujours d'après le Larousse, c'est vers la fin du $\mathrm{x} v \mathrm{i}^{\mathrm{e}}$ siècle qu'il commence à être utilisé en logique comme terme de spécialité avec la fonction «d'introduire la mineure d'un syllogisme». Il est intéressant de préciser que parallèlement, $O R$ conservait aussi sa valeur temporelle et que cette dernière s'est estompée peu à peu pour être pratiquement remplacée, de nos jours, par la valeur argumentative.

Le Robert dictionnaire analogique de la langue française définit OR comme, d'une part, un adverbe indiquant le temps et équivalant à «maintenant, présentement», et, d'autre part, une conjonction "marquant un moment particulier d'une durée ou d'un raisonnement». Il signale de plus un emploi de OR comme «terme de spécialité» dont il donne la définition suivante: «Or: particule introduisant la mineure d'un syllogisme, un argument ou une objection à une thèse.»

Cette dernière remarque, qui fait allusion aux valeurs argumentatives de ce connecteur, est particulièrement intéressante. En effet, les termes «argument» et «objection» suggèrent que le connecteur $O R$ n'a pas de valeur argumentative fixe et qu'il 
peut introduire aussi bien un énoncé co-orienté qu'un énoncé anti-orienté. Cette caractéristique met en évidence le rôle déterminant du contexte comme déclencheur argumentatif qui, comme je tenterai de le démontrer ci-dessous, guidera le traducteur dans la recherche d'un équivalent en espagnol.

\section{VALEURS ARGUMENTATIVES DE OR}

Dans une perspective pragmatique, Nina de Spengler (1980: 128), classe OR dans la catégorie «introducteur d'élément », c'est-à-dire, un morphème qui introduit une donnée nouvelle, ce qui correspond au schéma:

\section{p OR q}

où $\mathbf{p}$ et $\mathbf{q}$ sont deux arguments indépendants.

Cependant, elle ne formule aucune hypothèse quant à la valeur argumentative de ce connecteur. Aussi, je présenterai brièvement les possibilités argumentatives de $O R$ qui peuvent s'actualiser en contexte et qui seront approfondies lors de l'analyse du corpus.

Dans une optique argumentative, trois possibilités peuvent se présenter: q est anti-orienté, $\mathbf{q}$ est co-orienté, ou $\mathbf{q}$ est non-orienté par rapport à $\mathbf{p}$. Je précise que par non-orienté, je désigne les cas de figure où $\mathbf{q}$ ne sert ni à confirmer ni à infirmer $\mathbf{p}$, ce qui n'exclut pas qu'il puisse s'inscrire dans une stratégie argumentative globale orientée vers une certaine conclusion.

i) $O R$ introduit un argument anti-orienté

L'emploi de $O R$ comme introducteur d'argument anti-orienté est certainement le plus fréquent et correspond au schéma:

$$
\mathbf{p}(\longrightarrow \mathbf{r}) \text {. OR } \mathbf{q}(\longrightarrow \text { non-r })
$$

où l'on présente d'abord un premier énoncé $\mathbf{p}$ orienté vers une conclusion $\mathbf{r}$ et où on introduit ensuite un second énoncé q qui mène à une conclusion non-r.

ex. : Il n'y aura pas de progrès tangible dans la construction de l'Europe si celle-ci ne s'affirme pas avec force, courage et générosité à l'égard de l'extérieur. Or cette dimension est trop souvent négligée ou ignorée. (Delors 1992: 60)

ii) $O R$ introduit un argument co-orienté

Cet emploi de $O R$ est nettement moins fréquent que le précédent, et correspond au schéma :

$$
\mathbf{p}(\rightarrow \mathbf{r}) . \text { OR } \mathbf{q}(\rightarrow \mathbf{r})
$$

où un premier argument $\mathbf{p}$ orienté vers la conclusion $\mathbf{r}$ est présenté et un second argument $\mathbf{q}$ conforte $\mathbf{r}$.

ex.: L'enjeu, vous le connaissez. C'est celui du rapport quotidien de l'homme à son cadre de vie comme celui, millénaire, de la société à la nature. Or, dans ce combat devenu planétaire pour la préservation de l'environnement, l'Europe est en première ligne. (Delors 1992: 135)

La conclusion $\mathbf{r}$ peut être énoncée ou rester implicite. Lorsqu'elle est implicite, l'attribution d'une orientation argumentative à l'énoncé $\mathbf{q}$ est le résultat d'un processus d'inférence reposant sur les données contextuelles ou les connaissances de l'interlocuteur.

iii) $O R$ introduit un argument non-orienté

L'exemple le plus éloquent de l'emploi de $O R$ comme introducteur d'argument nonorienté est sans aucun doute le célèbre syllogisme: 
Tous les hommes sont mortels

Or Socrate est un homme

Donc Socrate est mortel.

où $\mathbf{p}$ est la proposition majeure, $\mathbf{q}$ la proposition mineure et $\mathbf{r}$ la conclusion.

Apparemment, cet exemple ne semble guère différent des précédents. En fait, la différence se situe au niveau du contenu sémantique des énoncés. En effet, l'énoncé $\mathbf{p}$ n'est pas sémantiquement orienté vers une conclusion déterminée.

Il est intéressant d'observer que, dans la version espagnole de ce syllogisme, tous les connecteurs disparaissent et la pensée passe d'une proposition à l'autre sans mot de liaison:

Todos los hombres son mortales

Socrates es un hombre

Socrates es mortal. (Fingermann 1972: 94)

Dans ce cas de figure, l'équivalence en espagnol semble donc être l'absence de connecteur que j'indiquerai par le signe $\varnothing$.

Cependant, l'emploi de $O R$ comme introducteur d'élément totalement nonorienté et intraduisible est rare. En fait, dans la plupart des textes étudiés, l'argument introduit par $O R$ est orienté soit vers une conclusion positive, soit vers une conclusion négative. Cette conclusion $\mathbf{r}$ sera le plus souvent explicite, surtout dans les textes scientifiques où la clarté de l'argumentation est un critère primordial.

\section{3. ÉQUIVALENTS ESPAGNOLS DE OR}

L'équivalent espagnol de $O R$ que donnent les dictionnaires bilingues (Larousse et Bordas) est ahora bien. Or, l'analyse du corpus révèle que, en contexte, le connecteur $O R$ est souvent traduit par pero, sin embargo, no obstante et $y$; je considère donc qu'il est intéressant de mentionner aussi les définitions de ces connecteurs.

Le Diccionario de la Real Academia Española et le Diccionario de Uso del Español de María Moliner donnent des connecteurs pero, ahora bien, sin embargo, no obstante, et $y$ les définitions suivantes:

pero: (del lat. per hoc) conjunción adversativa con que a un concepto se contrapone otro diverso o ampliativo del anterior. (DRAE)

pero: (del latin per hoc, por esto):

(1) Es una conjunción adversativa que expresa que lo que dice la oración a que afecta impide, justifica, compensa, contrarresta o atenúa lo dicho en la oración principal.

(2) También puede tener valor concesivo, expresando que lo que dice la oración afectada por «pero» se realiza a pesar de que sería natural otra cosa dado lo dicho en la oración principal.

(3) A veces, tiene sentido restrictivo. (M. Moliner)

ahora bien : locución conjuntiva adversativa. « esto supuesto o sentado.» (DRAE)

ahora bien : equivale a «pero» en expresiones como «Haz lo que quieras; ahora bien, atente a las consecuencias.» (M. Moliner)

no obstante: locución conjuntiva. Sin embargo. Sin que estorbe ni perjudique para una cosa. (DRAE)

no obstante: sin que la cosa de que se ha hablado constituya un obstáculo para lo que se dice luego (expresión concesiva). (M. Moliner) 
sin embargo: locución conjuntiva. No obstante. Sin que sirva de impedimento. (DRAE)

sin embargo: expresión adverbial concesivo-adversativa con que se alude a algo que, pudiendo causar o impedir cierta cosa que se expresa, no lo hace. (M. Moliner)

y (del lat. et) : conj. copulativa cuyo oficio es unir palabras o cláusulas en concepto afirmativo.(DRAE)

$\mathbf{y}$ (1) Es la conjunción coordinante copulativa por excelencia. Lo mismo que las otras de esta clase puede enlazar no solamente oraciones, sino elementos de una misma oración que hacen el mismo papel con respecto al verbo.

(2) A veces $y$ tiene sentido consecutivo, sobre todo cuando una de las oraciones es afirmativa y la otra negativa. "Conocía al capataz, y no se fiaba de él.»

(3) También tiene a veces sentido adversativo. «Está cansado y (y sin embargo) se empeña en seguir.»

(4) En algunos casos tiene valor concesivo. "Ande yo caliente y ríase (aunque se ría) la gente.» (M. Moliner)

Dans une perspective pragmatique, ces définitions permettent de classer les connecteurs espagnols en trois catégories:

1) Les connecteurs d'opposition-concession forts comme pero, sin embargo, no obstante, qui correspondent à l'anti-orientation claire et nette.

2) Les connecteurs d'opposition plus atténués comme ahora bien, et $y$ (acception no 3 , "sentido adversativo»), qui correspondent à une anti-orientation plus modérée.

3) Les connecteurs d'addition comme $y$ (acception du DRAE, « unir palabras o cláusulas en concepto afirmativo»), qui relient deux énoncés allant dans le même sens et peuvent donc véhiculer une notion de co-orientation.

Partant de ces définitions, on peut établir une première équivalence entre les connecteurs espagnols marquant l'opposition et le connecteur OR employé comme marqueur d'anti-orientation. Par contre, lorsque $O R$ est employé comme introducteur d'argument non-orienté ou introducteur d'argument co-orienté, la palette d'équivalents espagnols semble particulièrement réduite et seule l'analyse détaillée du contexte permettra de trouver un équivalent. C'est ce que je tenterai de démontrer à travers l'étude du corpus. À partir des trois valeurs de base de $O R$ (anti-orientation, introduction d'élément non-orienté et co-orientation), le corpus sera analysé de façon à établir, pour chaque valeur de base, des sous-divisions permettant de rendre compte des valeurs contextuelles du connecteur et des choix de traduction auxquels elles conduisent.

\section{CATÉGORISATION ET TRADUCTION DE OR}

\subsection{Introduction d'argument anti-orienté}

Bien qu'il s'agisse de l'emploi de $O R$ le plus facile à identifier, et que dans la plupart des textes étudiés la traduction de cette valeur ne fasse pas problème, je présenterai ci-dessous les divers cas de figure correspondant à «or: introducteur d'élément antiorienté» car ils permettent de cerner les éléments qui entrent en jeu et qui peuvent servir de base à une catégorisation des emplois de ce morphème. 


\subsubsection{L'invalidation absolue ou réfutation}

L'invalidation absolue ou réfutation clôt toute possibilité de donner une suite à l'argumentation. Elle correspond au schéma:

$$
\begin{array}{ll}
\mathbf{p} \rightarrow \mathbf{r . ~ O r} & \text { non-p }(\rightarrow \text { non-r }) \\
& \text { non-r }(\rightarrow \text { non-p) } \\
& \text { non }(\mathbf{p} \rightarrow \mathbf{r})
\end{array}
$$

où $\mathbf{p}$ est un argument qui conduit à la conclusion $\mathbf{r}$ et l'argument introduit par $O R$ est la négation catégorique soit de $\mathbf{p}$, soit de $\mathbf{r}$, soit de la relation entre $\mathbf{p}$ et $\mathbf{r}$.

Dans les textes scientifiques, l'invalidation absolue apparait souvent comme l'expression linguistique de l'application du modus tollens (si A alors B - B est faux donc A est faux). Un tel raisonnement peut se dérouler: a) dans un univers hypothétique, c'est ce que j'appellerai «invalidation d'une hypothèse », ou b) dans l'univers réel, c'est ce que j'appellerai «invalidation d'une condition».

\section{a) Invalidation d'une hypothèse}

L'invalidation d'une hypothèse est typiquement marquée, du point de vue syntaxique, par l'emploi de certaines formes verbales et peut être représentée ainsi:

dans un univers hypothétique: Si $\mathbf{p}$ (imparfait ind.) $\rightarrow \mathbf{r}$ (conditionnel)

$O R$

dans l'univers réel: non-r (présent ind.) (- DONC $\rightarrow$ non-p)

illustré par l'exemple ${ }^{2}$ suivant:

(1a) Comme les petites rides contiennent moins de matériaux que les grandes, elles progressent plus vite dans le sens du vent et ont donc tendance à rattraper les rides plus grandes et à fusionner avec elles. Ainsi, les grandes rides croissent aux dépens des petites et, en général, les différences de tailles entre les rides s'estompent. Cependant, si ce processus de propagation des rides se révélait exact, on pourrait s'attendre à ce qu'il ne subsiste, à la fin, plus qu'une seule ride géante. Or il n'en est rien. [Fin du paragraphe] (L.R. 247: 1180)

(1b) Como las ondulaciones pequeñas contienen menos material que las grandes, avanzan más aprisa en el sentido del viento $y$, por tanto, tienen tendencia a alcanzar las ondulaciones mayores y a fusionarse con ellas. Por tanto, las ondulaciones grandes crecen a expensas de las pequeñas $y$, en general, las diferencias de tamaños entre ondulaciones van desapareciendo. Sin embargo, si este proceso de propagación de las ondulaciones demostrara ser exacto, cabría esperar que, al final, únicamente subsistiera una sola ondulación gigante.

Pero no es así. (M.C. 130: 1070)

En (1a), le premier énoncé $\mathbf{p}$ «si ce processus se révélait exact» reprend anaphoriquement le processus «les petites rides rattrapent les grandes et fusionnent avec elles» et, par le créateur d'univers ${ }^{3}$ «si », sert de point d'ancrage à l'hypothèse.

Le résultat $\mathbf{r}$, marqué syntaxiquement par le verbe au conditionnel, est une implication logique de $\mathbf{p}$ : "les petites rides disparaîtraient et il ne subsisterait qu'une ride géante».

Le connecteur $O R$ introduit un fait réel observable qui réfute $\mathbf{r}$, et par là même invalide l'hypothèse $\mathbf{p}$ et clôt l'argumentation. Cette valeur de conclusion anti-orientée est soulignée, de plus, par le fait que l'énoncé introduit par $O R$ marque la fin du paragraphe. 
Dans l'exemple (2a), on observe que l'invalidation est aussi totale que dans l'exemple (1a); cependant l'énoncé introduit par $O R$ n'a pas en (2a) la valeur de conclusion qu'il a en (1a) puisqu'il est suivi d'une conclusion explicite, introduite par un connecteur consécutif «donc» et par un élément lexical, «il faut conclure», qui exprime clairement la clôture.

(2a) C'est donc que le système nerveux, lui, a une telle partie arithmétique fonctionnant à un niveau de précision élevé.

Or cette hypothèse est impossible: il faut donc conclure que le système nerveux diffère de l'ordinateur sur ce point, en parvenant à accomplir sa tâche extraordinairement compliquée à un faible niveau de précision, tout en ayant un niveau élevé de fiabilité. (L.R. 252: 343)

(2b) De ello se deduciría que el sistema nervioso tiene una parte aritmética de esta naturaleza que funciona con un alto nivel de precisión.

Ahora bien, esta hipótesis es imposible: por tanto se llega a la conclusión de que el sistema nervioso difiere en este punto del ordenador y consigue cumplir su tarea, extraordinariamente complicada, a un bajo nivel de precisión, pero teniendo un alto nivel de fiabilidad.

(M.C. 135: 477)

En (2a), l'étude du co-texte permet de préciser que l'argument $\mathbf{p}$ «le système nerveux a une telle partie arithmétique fonctionnant à un niveau de précision élevé » apparaît comme la conclusion de tout un paragraphe qui présente des arguments en faveur d'une analogie éventuelle entre le cerveau et l'ordinateur. Il s'agit cependant de la conclusion d'une hypothèse, ce qui, dans le texte français, se traduit par la reprise «c'est donc que» qu'on pourrait paraphraser par «d'après ce que l'on vient d'exposer» et qui donne à l'argument une validité relative, dépendante du contexte. Cette nuance a été d'ailleurs parfaitement perçue par le traducteur espagnol qui a opté pour l'expression «de ello se deduciría». De cet argument $\mathbf{p}$ on peut tirer la conclusion $\mathbf{r}$ à partir de l'expression anaphorique «telle» qui a comme référent l'ordinateur et qui peut être paraphrasée par «comme celle d'un ordinateur», d'où le résultat implicite $\mathbf{r}$ : «le système nerveux ressemble à un ordinateur» qui est invalidé par l'énoncé introduit par $O R$.

L'emploi de pero en (2b) ne poserait aucun problème puisque c'est un connecteur qui marque l'invalidation et qui peut introduire aussi bien une conclusion qu'un argument. Par contre, l'emploi de ahora bien en (1b) qui donnerait un énoncé du type:

(?) Ahora bien esto no es así. (Fin du paragraphe)

paraîtrait plutôt surprenant puisqu'on s'attend après ahora bien, à une pause — c'està-dire une virgule dans un texte écrit - suivie de la présentation d'un argument ou de tout un mouvement discursif ${ }^{4}$.

La différence entre l'emploi de pero et celui de ahora bien vient donc respectivement, dans ce cas, de la valeur de clôture et de poursuite que présentent ces deux connecteurs.

Cette valeur de poursuite apparaît plus clairement dans certains contextes.

(3a) La géométrie à deux dimensions que représentent les plans $\mathrm{CuO} 2$ est à l'origine d'une autre théorie, celle des «anyons» [...]. L'immense mérite de cette théorie est d'avoir proposé aux expérimentateurs des prédictions réfutables. L'une d'elles est que les anyons, à la manière d'un champ magnétique, brisent la symétrie gauche-droite. Or 
on sait polariser la lumière de sorte que le vecteur champ électrique décrive, lors de la propagation de l'onde, une hélice gauche ou droite [...]. (c'est-à-dire s'enroule dans un sens ou dans l'autre autour de la direction de propagation); on parle alors de lumière "gauche» ou «droite». [...] L'expérience, simple dans son principe, a été tentée dans plusieurs laboratoires. [...] Son résultat est totalement négatif, ce qui signifie que les anyons ne sont sans doute pas une représentation appropriée de l'état électronique des plans cuivre-oxygène. (L.R. 240: 185)

(3b) La geometría de dos dimensiones de los planos $\mathrm{CuO} 2$ está en el origen de otra teoría, la de los "anyones» [...]. Esta teoría tiene el mérito inmenso de haber propuesto a los experimentadores unas predicciones refutables. Una de ellas es que los anyones, a la manera de un campo magnético, rompen la simetría izquierda-derecha. Ø Sabemos polarizar la luz de tal modo que el campo vector eléctrico, durante la propagación de la onda, describa una hélice levógira o dextrógira (es decir que se arrolle en uno u otro sentido en torno a la dirección de propagación); se habla entonces de luz «levógira»o "dextrógira». [...]El experimento, simple en su fundamento, ha sido intentado en varios laboratorios. [...]Su resultado es totalmente negativo, lo cual significa que los anyones, muy probablemente, no son una representación apropiada del estado electrónico de los planos cobre-oxígeno. (M.C. $123: 333)$

Le mouvement discursif représenté en (3a) est particulièrement complexe. Une hypothèse $\mathbf{p}$ «les anyons, à la manière d'un champ magnétique, brisent la symétrie gauche-droite» est introduite et expliquée dans un premier énoncé, puis un second énoncé introduit par $O R$ présente l'élément nouveau, à savoir l'expérience de la polarisation de la lumière. À ce moment précis du texte, on ne sait pas si cette expérience va valider ou invalider l'hypothèse. C'est pourquoi le traducteur a résisté à l'emploi de pero, et a supprimé le connecteur. Cependant, si l'on veut éviter que le lecteur perde le fil de l'argumentation, il est préférable de marquer clairement le point de blocage de la trajectoire, c'est-à-dire, dans ce cas, le point où commence la réfutation de l'hypothèse. Dans un tel contexte, l'expression ahora bien, peut fort bien marquer ce début de mouvement d'invalidation.

\section{b) Invalidation d'une condition}

Dans ce que je désigne comme «invalidation d'une condition", le raisonnement de type modus tollens s'applique à une situation réelle. Lorsque le morphème «si » apparaît, il marque donc non plus une hypothèse mais une éventualité, ce qui peut être représenté de la façon suivante:

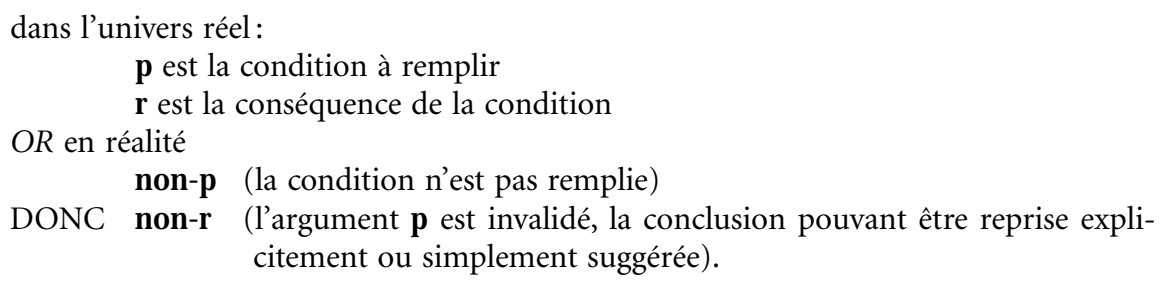

L’opposition s'établit donc entre une condition présentée comme nécessaire pour qu'un certain fait se produise, et la constatation qu'une telle condition n'est pas remplie.

(4a) La fermeture latérale de l'espace lingotière constitue aussi un problème d'un haut niveau de difficulté technologique: les plaques sont généralement appuyées sur les 
cylindres qui tournent. Pour que soit assurée une étanchéité complète au métal liquide, les jeux entre plaques et cylindres ne doivent pas excéder le dixième de millimètre. $\mathbf{O r}$ les cylindres se déforment de plusieurs millimètres, latéralement et diamétralement, pendant une coulée. Les plaques doivent en outre être aussi réfractaires que possible, afin d'éviter toute solidification qui, lorsqu'elle se produit, induit des défauts sur les bandes. (L.R. 240: 157)

(4b) El cierre lateral del espacio de la lingotera constituye también un problema de alto nivel de dificultad tecnológica: las placas suelen apoyarse en los cilindros que giran. Para que la estanqueidad respecto al metal líquido, sea completa, el juego entre placas y cilindros no ha de superar la décima de milimetro. Pero durante una colada los cilindros se deforman varios milímetros, lateralmente y diametralmente. Además, las placas tienen que ser lo más refractarias posible para evitar toda solidificación que, de producirse, daría lugar a defectos en la chapa. (M.C. 123: 315)

En (4a), l'ordre des termes est inversé et l'implication qui sous-tend le raisonnement apparaît sous forme de proposition de but; ainsi au lieu de:

$$
[\text { si } \mathbf{p} \longrightarrow \mathbf{r}]
$$

la condition est présentée sous la forme

$$
\text { [pour que } \mathbf{r}, \mathbf{p} \text { ] }
$$

mais la relation logique de base est la même.

En (4a), la conclusion implicite non-r (l'espace lingotière n'est pas étanche) est en quelque sorte annoncée dès le début par l'expression «un problème d'un haut niveau de difficulté technologique». Les énoncés suivants expliquent donc pourquoi ce problème se présente et cette explication est fondée sur une opposition entre l'objectif poursuivi et le résultat obtenu. On pose donc $\mathbf{p}$ «les jeux entre plaques et cylindres ne doivent pas excéder le dixième de millimètre» comme la condition permettant d'atteindre l'objectif, et on lui oppose non-p (les cylindres se déforment) qui représente un obstacle et renvoie à la conclusion non-r déjà annoncée.

\subsubsection{L'invalidation partielle}

Dans l'invalidation partielle, il n'y a pas négation de la totalité de l'énoncé précédent, mais négation de l'un des éléments de cet énoncé. Il s'agit d'expliquer pourquoi certains phénomènes qui se produisaient dans des conditions déterminées ne se produisent plus lorsqu'il y a modification de ces conditions. L'invalidation peut alors porter sur différents aspects : a) changement dans le temps, b) restriction, c) invalidation d'un terme de $\mathbf{p}$.

a) L'invalidation due à un changement dans le temps correspond au schéma:

$$
\text { avant } \mathbf{t}, \mathbf{p}(\longrightarrow \mathbf{r}) \text {. OR depuis } \mathbf{t}, \mathbf{q} \longrightarrow \text { non-r. }
$$

L'opposition entre $\mathbf{p}$ et $\mathbf{q}$ est axée sur le moment $\mathbf{t}$, qui représente le blocage et le renversement de situation. À ce niveau, on observe qu'un lien étroit subsiste entre la valeur temporelle étymologique de OR et sa valeur argumentative.

(5a) Cette stratégie (production sidérurgique concentrée autour de quelques grands sites) s'est traduite par la réalisation d'usines intégrées géantes qui manquent parfois de souplesse pour s'adapter aux fluctuations du marché. Or, depuis deux décennies envi- 
ron, des recherches sont menées pour modifier ces outils de production. Elles ont conduit à la conception de procédés entièrement nouveaux, qui permettent de fabriquer directement, sans étape de laminage, des tôles minces. (L.R. 240 : 151)

(5b) Esta estrategia se ha traducido en la realización de fábricas integradas gigantes, que a veces carecen de la flexibilidad necesaria para adaptarse a las fluctuaciones del mercado. No obstante, desde hace unos dos decenios, se realizan investigaciones destinadas a modificar estos instrumentos de producción. Dichas investigaciones han llevado a idear unos procesos totalmente nuevos que permiten fabricar chapas delgadas directamente, sin etapa de laminado. (M.C. 123: 309)

L'exemple (5a) présente un fait réel, $\mathbf{p}$ «la réalisation d'usines intégrées » qui appartient au passé (antérieur au moment $\mathbf{t}$ ). Le connecteur $O R$, combiné à l'expression temporelle «depuis deux décennies environ», indique une opposition entre $\mathbf{p}$, «les usines intégrées », qui supposent des procédés peu flexibles, et $\mathbf{q}$, «les procédés beaucoup plus flexibles », qui sont introduits actuellement.

La traduction de $O R$ par un terme invalidant comme no obstante est adéquate dans ce cas parce que l'argument introduit par OR renforce une invalidation déjà amorcée dans l'énoncé précédent par des éléments lexicaux. En effet, l'expression "qui manquent parfois de souplesse» exprime la constatation d'une carence qui suggère la recherche d'une solution, c'est-à-dire qui annonce de façon implicite l'invalidation de $\mathbf{p}$; l'expression "fluctuations du marché» indique par ailleurs que cette carence s'applique à la situation présente puisque, en l'absence de spécification temporelle, «du marché» est interprété comme un terme anaphorique équivalent à «actuellement». Le connecteur $O R$ introduit donc une reprise de cet implicite et l'expression temporelle «depuis deux décennies environ » est la charnière qui marque ce renversement de situation.

\section{b) La restriction}

Dans l'invalidation par restriction, un premier argument $\mathbf{p}$ est présenté et un second argument $\mathbf{q}$ introduit les conditions dans lesquelles $\mathbf{p}$ est invalidé. Le schéma correspondant à cette invalidation sera:

$$
\text { p. OR q (= dans certaines conditions) } \longrightarrow \text { non-p. }
$$

(6a) Dans l'état supraconducteur, selon la théorie BCS, les électrons qui conduisent le courant électrique sont groupés deux à deux en "paires de Cooper», formant un superfluide qui s'écoule sans résistance. Or le courant confère aux paires de Cooper une énergie cinétique qui, $\mathbf{s i}$ elle excède l'énergie de désappariement, détruit les paires — et avec elles la supraconductivité — et restaure l'effet Joule (dissipation d'énergie électrique en chaleur). (L.R. 240: 183)

(6b) En el estado supraconductor, según la teoría BCS, los electrones que conducen la corriente eléctrica van de dos en dos en "pares de Cooper", formando un superfluido que se mueve sin resistencia. Pero la corriente confiere a los pares de Cooper una energía cinética que, cuando supera la energía de acoplamiento, destruye los pares, y con ellos la super-conductividad, y restaura el efecto Joule (disipación de energía eléctrica en forma de calor). (M.C. 123: 331)

En (6a), un premier énoncé $\mathbf{p}$ présente un phénomène «dans l'état supraconducteur, les paires de Cooper forment un superfluide qui s'écoule sans résistance», et un second énoncé $\mathbf{q}$ présente une condition «si l'énergie cinétique dépasse un certain 
niveau », qui empêche que ce phénomène se produise (la superconductivité est détruite). L'argument introduit par $O R$ marque donc l'invalidation de $\mathbf{p}$, et spécifie les conditions dans lesquelles elle se réalise.

Il est intéressant de noter que le morphème «si » est celui qui marque le plus clairement cette notion de restriction (dans certaines conditions), mais qu'il n'est certainement pas le seul. Certaines unités lexicales ont ainsi la particularité de configurer une situation déterminée en indiquant l'absence ou la présence de certains éléments; on relève par exemple dans le corpus les expressions : en présence de..., en l'absence de..., sans..., etc.

\section{c) Invalidation d'un terme seulement}

De façon générale, dans ce cas de figure, un argument $\mathbf{p}$, qui établit une relation entre deux termes $\mathbf{p} \mathbf{1}$ et $\mathbf{p 2}$, est suivi d'un énoncé introduit par $O R$ qui invalide l'un des termes seulement, ce qui peut être représenté par le schéma:

$$
\mathbf{p}(\mathbf{p 1}+\mathbf{p 2}) . \text { OR q (= non-p1) }
$$

où le fait d'invalider $\mathbf{p} 1$ n'invalide pas $\mathbf{p}$, comme le montre l'exemple suivant:

(7a) Cette diminution durable de l'efficacité d'une synapse à glutamate apparaît au niveau des synapses entre de grandes cellules du cervelet, les cellules de Purkinje, et les axones des cellules en grains (fibres parallèles). Or les cellules de Purkinje sont parmi les rares neurones du cervelet où la No-synthase n'a pu être mise en évidence à ce jour. Il faut donc imaginer l'intervention d'un autre type de cellules dans la production du monoxyde d'azote. (L.R. $240: 241$ )

(7b) Esta diminución duradera de la eficacia de una sinapsis con glutamato aparece a nivel de las sinapsis entre grandes células del cerebelo, las células de Purkinje, y los axones de las células en granos (fibras paralelas). Sin embargo, las células de Purkinje son unas de las raras neuronas del cerebelo en las que, hasta ahora, no ha podido descubrirse la No-sintasa. Por consiguiente, hay que pensar en la intervención de otro tipo de células para la producción del monóxido de nitrógeno. (M.C. 123: 365)

En (7a), le premier argument $\mathbf{p}$ établit l'apparition d'un phénomène attribué à l'interaction de deux éléments $\mathbf{p} \mathbf{1}$ «les cellules de Purkinge» et $\mathbf{p} \mathbf{2}$ «les axones des cellules en grains ». L'énoncé introduit par or, invalide en fait l'attribution du phénomène à p1, mais n'invalide pas $\mathbf{p}$ (l'apparition du phénomène), ce qui conduit à la conclusion qu'un autre type de cellules doit entrer en jeu.

On observe donc qu'en ce qui concerne la traduction de $O R$ comme morphème marquant l'invalidation partielle, les connecteurs espagnols sin embargo et no obstante conservent le caractère nuancé de l'invalidation alors que pero semble souligner davantage la valeur d'invalidation en elle-même. Ahora bien peut aussi être employé lorsque l'opération d'invalidation que déclenche l'énoncé $\mathbf{q}$ repose sur un mouvement plus long. Le choix du connecteur devra donc reposer à la fois sur la nature de l'invalidation présentée et sur les caractéristiques du mouvement discursif qui l'englobe.

\subsection{Introduction d'argument non-orienté}

Du point de vue de la traduction, je signale que c'est dans cette catégorie que se produit le plus grand nombre d'erreurs ou de traductions maladroites. Dans la plu- 
part des cas on observe que, dans la traduction espagnole, le connecteur est simplement omis, alors que l'explicitation des relations textuelles entre l'argument nouveau introduit par $O R$ et les autres éléments du cotexte permet de trouver des équivalents en espagnol, ce qui a pour conséquence d'éviter l'aspect de message télégraphique que produit la simple juxtaposition.

Avant d'aborder l'étude de cette catégorie, je rappelle que par le terme «argument non-orienté » j'entends un argument dont la fonction n'est pas d'invalider ou de valider directement l'argument précédent, mais d'apporter une information permettant d'arriver à une conclusion. De ce point de vue, le schéma de $O R$ comme introducteur d'élément non-orienté semble se rapprocher du schéma du syllogisme: un premier énoncé $\mathbf{p}$ est présenté, auquel vient s'ajouter un second argument $\mathbf{q}$ introduit par $O R$, la somme de ces deux arguments conduisant à une conclusion $\mathbf{S}$. Ce qui peut être représenté de la façon suivante:

\section{p. $O R$ q. Donc $\mathbf{s}$}

où $\mathbf{S}$ n'est ni la conséquence de $\mathbf{p}$, ni la conséquence de $\mathbf{q}$, mais le résultat de la combinaison de $\mathbf{p}$ et $\mathbf{q}$.

Dans cette catégorie, je distinguerai deux types d'arguments: a) les arguments additionnels sans ancrage temporel, et b) les arguments additionnels avec ancrage temporel.

\subsubsection{Arguments additionnels sans ancrage temporel}

Les arguments additionnels sans ancrage temporel sont des arguments qui sont simplement ajoutés à un premier argument pour conduire à une conclusion et qui correspondent au schéma général d'introduction d'argument non-orienté présenté ci-dessus. Ce cas de figure peut être illustré par l'exemple ci-dessous :

(8a) Comme les photons qui constituent la lumière, les atomes sont simultanément des corpuscules et des ondes. Or l'une des principales caractéristiques des ondes est leur faculté d'interférer. Le cliché montre ainsi des franges d'interférences analogues à celles obtenues avec de la lumière blanche [...]. (L.R. 247: 1135)

(8b) Como los fotones que constituyen la luz, los átomos son simultáneamente corpúsculos $y$ ondas. $\varnothing$ Una de las principales características de las ondas es su capacidad de interferir. La foto muestra franjas de interferencia análogas a las que se obtienen con luz blanca [...]. (M.C. 130: 1009)

En (8a), un premier argument $\mathbf{p}$ «les atomes sont simultanément des corpuscules et des ondes» est suivi d'un second argument $\mathbf{q}$ «l'une des principales caractéristiques des ondes est leur faculté d'interférer» qui apporte un élément nouveau non-orienté par rapport à $\mathbf{p}$, permettant d'arriver à la conclusion $\mathbf{S}$ «le cliché montre ainsi des franges d'interférences » où "ainsi» a une valeur consécutive/conclusive semblable à celle de $«$ donc $»^{5}$.

On observe donc que dans la version espagnole de ce passage, tout comme dans la version espagnole du syllogisme, les connecteurs ont disparu et les énoncés ont été juxtaposés. Cependant, si cette absence de liaison est habituelle dans un syllogisme qui s'apparente à un langage formel, elle l'est moins en langage naturel où elle donne une impression de communication télégraphique. En fait, pour la traduction de $O R$, 
une légère réorganisation passant par l'introduction d'une conjonction de coordination et une modification de la ponctuation peuvent apporter une solution. Quant à «ainsi », il peut être rendu par así pues:

[Como los fotones que constituyen la luz, los átomos son simultáneamente corpúsculos y ondas, $\mathbf{y}$ una de las principales características de las ondas es su capacidad de interferir. Así pues, la foto muestra franjas de interferencia análogas a las que se obtienen con luz blanca]

Cette mise en relation permet de renforcer la cohésion du texte, même s'il est parfois nécessaire d'apporter de légères modifications syntaxiques.

L'exemple (8) correspond à l'addition d'un élément à un autre, mais [or q] peut aussi être employé pour introduire un argument dans une série, comme le montre l'exemple (9); on aura alors un schéma du type:

\section{A. Connecteur p. Or q. Connecteur. B $\rightarrow \mathbf{s}$}

9a) Si le principe de ce type d'appareils est relativement simple du point de vue de la création et de la détection des rayons gamma caractéristiques, il n'en est pas de même du traitement de l'information fournie par les détecteurs. En effet, l'amplitude des signaux est liée à l'énergie des photons émis. Or la forme du spectre en énergie est liée à la nature du milieu analysé. Le temps de détection est également important. Ce sont donc de nombreux paramètres qui doivent être analysés simultanément. (L.R. 247:130)

(9b) El principio de este tipo de aparatos es bastante simple desde el punto de vista de la creación y la detección de los rayos gamma característicos, pero no se puede decir lo mismo del tratamiento de la información dada por los receptores. En efecto, la amplitud de las señales está ligada a la energía de los fotones emitidos. Pero la forma del espectro energético esta relacionada con la naturaleza del medio analizado. El tiempo de detección también es importante. (M.C. $130: 1062$ )

En (9a), la relation exprimée par $O R$ est assez complexe, car elle marque l'intégration de l'argument $\mathbf{q}$ dans une suite de trois arguments qui s'ajoutent et conduisent à une conclusion, le schéma de cet exemple étant:

$$
\begin{aligned}
& A=\text { le traitement de l'information fournie par les détecteurs est complexe } \\
& \text { En effet: raison 1: l'amplitude des signaux est variable } \\
& \text { Or: } \quad \text { raison 2: la forme du spectre est aussi variable } \\
& \text {...également raison 3: le temps de détection est important }
\end{aligned}
$$

L'énoncé $\mathbf{A}$ présente un problème «la complexité du traitement de l'information » et les énoncés $\mathbf{p}, \mathbf{q}$ et $\mathbf{B}$ apportent une série de raisons qui justifient $\mathbf{A}$ en expliquant sa complexité. La traduction de $O R$ par pero est à éviter dans ce cas, car la fonction de $O R$ est de marquer non pas un mouvement d'anti-orientation mais l'insertion d'un argument dans une séquence anti-orientée. Aussi, une traduction par un connecteur comme además, qui suggère non seulement l'addition mais aussi la pluralité des éléments semble rendre plus fidèlement la relation établie par $O R$.

\subsubsection{Arguments avec ancrage temporel}

Afin d'éviter les termes «diachronique» et «synchronique» très utilisés en linguistique et donc chargés de connotations dans ce domaine, j’ai volontairement emprunté au vocabulaire technologique les termes «synchrone», utilisé pour désigner des phé- 
nomènes révolutifs qui s'accomplissent dans la même période de temps, et son antonyme «asynchrone».

\section{a) Arguments synchrones}

Il s'agit de deux arguments qui se développent parallèlement dans un même segment temporel $\mathbf{t}$, et qui se combinent pour donner une conclusion, ce que l'on peut représenter de la façon suivante:

$$
\mathbf{p} \text { (dans un temps } \mathbf{t} \text { ). OR } \mathbf{q} \text { (dans un même temps } \mathbf{t} \text { ) } \rightarrow \mathbf{s}
$$

(10a) La découverte, en 1977, de vastes communautés animales en mer profonde constitue pour les géologistes océanographes l'événement majeur de ces quinze dernières années. Ces populations vivent à des profondeurs variables, de 1500 à $2000 \mathrm{~m}$ dans les sites d'hydrothermalisme actif de la dorsale du Pacifique Oriental, de 3800 à $5900 \mathrm{~m}$ dans les zones de subduction du Japon. Or les recherches menées depuis le début des années quatre-vingts montrent que les processus de symbiose qui assurent la pérennité de ces communautés existent également chez des invertébrés du littoral, notamment des mollusques bivalves, par exemple sur les côtes de la Bretagne. Ainsi, l'étude de ces mollusques nous apporte de nombreux renseignements sur les phénomènes biologiques existant sous la mer, en des lieux peu accessibles. (L.R. $240: 242$ )

(10b) El descubrimiento, en 1977, de amplias comunidades animales en mares profundos constituye para los biólogos oceanógrafos el acontecimiento más importante de estos últimos quince años. Estas poblaciones viven a profundidades variables, de 1500 a 2700 metros en los lugares con un hidrotermalismo activo de la dorsal del Pacífico Oriental, y de 3800 a 5900 metros en la zonas de subducción de Japón. Y las investigaciones realizadas desde el principio de los años ochenta demuestran que los procesos de simbiosis que aseguran la perennidad de estas comunidades también existen en invertebrados del litoral, en especial en moluscos bivalvos, por ejemplo en las costas de Bretaña. Así el estudio de estos moluscos nos aporta numerosas informaciones sobre los fenómenos biológicos que existen bajo el mar, en lugares poco accesibles. (M.C. 123: 366)

L'exemple (10a) présente deux faits $\mathbf{p}$ et $\mathbf{q}$ qui se déroulent dans un espace géographique différent mais pratiquement dans un même espace temporel puisque $\mathbf{p}$ (l'intérêt de l'étude de ces communautés animales en mer) surgit en 1977 et continue pendant environ quinze ans, et $\mathbf{q}$ (étude des invertébrés du littoral) débute au début des années quatre-vingts et se poursuit depuis lors (je précise que l'article date de 1992). Il y a donc addition d'éléments simultanés que le traducteur a rendu par $y$, terme passe-partout en l'absence de marques d'anti-orientation, mais que l'on pourrait aussi traduire par un terme comme paralelamente, qui souligne davantage l'idée de simultanéité.

\section{b) Arguments asynchrones}

Dans ce cas de figure, les arguments présentés se situent à des périodes différentes, ce qui correspond au schéma:

$$
\left.\mathbf{p} \text { (dans un temps } \mathbf{t}) \text {. OR } \mathbf{q} \text { (dans un temps } \mathbf{t}^{\prime}\right) \rightarrow \mathbf{s}
$$

(11a) (résumé du contexte: En 1980, les chercheurs découvrent l'existence dans les vaisseaux sanguins d'un facteur relaxant qu'ils baptisent EDRF qui agit comme messager intracellulaire.)

Or en 1987, l'EDRF a été identifié par l'équipe de S. Moncada, des Laboratoires 
Welcome, en Grande-Bretagne, comme étant le monoxyde d'azote. L'idée que ce gaz pourrait être également un messager intercellulaire dans le système nerveux central provient de travaux effectués par le laboratoire de J. Garthwaite, à l'université de Liverpool, en Grande-Bretagne. En 1988, cette équipe montre... (L.R. 240: 240)

(11b) (En 1980, los investigadores demostraban la existencia en los vasos sanguíneos de un factor relajante que denominaban EDRF.)

Ahora bien, en 1987, el EDRF fue identificado por el equipo de S. Moncada, de los laboratorios Welcome de Gran Bretaña, como el monóxido de nitrógeno. La idea de que este gas podría ser también un mensajero intercelular en el sistema nervioso central arranca de los trabajos llevados a cabo por el laboratorio de J. Garthwaite, de la universidad de Liverpool, en Gran Bretaña. (M.C. 123: 364)

L'exemple (11a) présente d'une part un argument $\mathbf{p}$ qui pose l'existence d'une substance agissant dans les vaisseaux sanguins comme messager intracellulaire et d'autre part un argument $\mathbf{q}$ qui révèle que cette substance est le monoxyde d'azote. On observe que l'argument $\mathbf{p}$ se situe en 1980 alors que l'argument $\mathbf{q}$ se situe en 1987. Il y a donc un décalage dans le temps, souligné par $O R$ dont la valeur argumentative semble éclipsée par la valeur temporelle; mais la fonction de $O R$ est en fait plus complexe. Placé en début de paragraphe, ce connecteur a aussi un rôle de marqueur de structuration du texte puisqu'il marque le point d'articulation entre deux arguments qui semblent opposés: l'EDRF est un messager intracellulaire et l'EDRF est un messager intercellulaire. Dans ce cas, l'expression espagnole ahora bien qui marque à la fois ce paradoxe et la limite entre ce qui appartient au passé et ce qui appartient au présent semble être une bonne solution.

\subsection{Introduction d'un argument co-orienté}

La dernière valeur de $O R$ que j'examinerai est celle d'introducteur d'argument coorienté qui correspond aux cas où l'énoncé introduit par $O R$ vise à renforcer ou à confirmer un premier argument. Dans cette catégorie, je distinguerai aussi plusieurs sous-catégories en fonction du type de relation qui est établi entre $\mathbf{p}$ et $\mathbf{q}$, à savoir : $\mathbf{a})$ relation causale entre argument et conclusion, et $\mathbf{b}$ ) validation absolue de l'argument présenté en $\mathbf{p}$.

\subsubsection{Relation causale entre argument et conclusion}

Dans ce cas de figure, un premier énoncé présente l'affirmation d'une relation entre $\mathbf{p}$ et $\mathbf{q}$ sous la forme [ $\mathbf{p}$ a la propriété $\mathbf{q}$ ] et l'énoncé introduit par $O R$ souligne l'importance de $\mathbf{q}$ et, par là même, revalorise $\mathbf{p}$, ce qui peut être représenté par le schéma:

$$
\text { p (q). OR q. Alors } \mathbf{p}
$$

dont la lecture est:

$$
\begin{aligned}
& \mathbf{p} \text { a la propriété } \mathbf{q} \text {. } \\
& O R, \\
& \text { parce que } \mathbf{q} \text { est important } \rightarrow \mathbf{p} \text { est revalorisé }
\end{aligned}
$$

et que j'illustrerai par l'exemple suivant: 
(12a) Les perspectives [applications des couches minces] sont donc prometteuses. [...] C'est également dans les couches minces qu'on enregistre aujourd'hui les densités de courant (intensité par centimètre carré de section) les plus élevées. Or de fortes densités de courant sont un préliminaire indispensable aux applications ultérieures qui nécessitent des courants électriques importants (moteurs, transformateurs, transport du courant,...) ou des champs magnétiques intenses (imagerie médicale par résonance magnétique). (L.R. 240: 183)

(12b) Estas perspectivas [de aplicación de las capas débiles] son prometedoras. [...] Es también en las capas débiles donde hoy se registran las mayores densidad de corriente (intensidad por centímetro cuadrado de sección). Pero las fuertes densidades de corriente sont un requisito indispensable para las aplicaciones ulteriores que requieren corrientes eléctricas importantes (motores, transformadores, transporte de corriente...) o campos magnéticos intensos (imaginerías médica por resonancia magnética). (M.C. 123: 331)

En (12a), l'auteur présente dans l'énoncé $\mathbf{p}$ un élément (les couches minces) qui a la propriété q (les fortes densités de courant), et d'autre part un argument OR q (les fortes densités de courant sont indispensables) qui souligne l'importance de cette propriété $\mathbf{q}$ et, de ce fait, revalorise $\mathbf{p}$.

La traduction par pero (en 12b) est évidemment à éviter, puisqu'elle introduit une valeur d'anti-orientation qui est le contraire de la valeur de co-orientation/ confirmation présente dans ce texte. L'équivalent espagnol serait donc à rechercher parmi des expressions de confirmation/insistance, comme justamente ou precisamente.

\subsubsection{Validation absolue}

Dans le cas de la validation absolue, $\mathbf{p}$ est présenté comme un argument hypothétique et l'argument $\mathbf{q}$, introduit par OR, sert à valider l'hypothèse $\mathbf{p}$, selon le schéma:

$$
\text { p ? OR q. alors } \mathbf{p}
$$

qu'on peut paraphraser par:

$$
\begin{aligned}
& \mathbf{p} \text { (hypothèse). } \\
& \text { OR } \mathbf{q} \text { (confirme la validité de } \mathbf{p}) \longrightarrow \mathbf{p} \text { est validé. }
\end{aligned}
$$

L'exemple (13a) est intéressant parce qu'il utilise l'implicite d'un façon particulièrement efficace. Afin de mieux comprendre le sens de ce passage, je précise qu'il s'agit d'un article dont le titre est «L'optique atomique» et dont le but est de démontrer que l'utilisation des atomes en optique suppose une amélioration substantielle des instruments utilisés dans ce domaine.

(13a) La solution idéale serait d'utiliser, selon les mêmes principes qu'un microscope optique ou électronique, des particules ayant simultanément une faible énergie cinétique et une courte longueur d'onde. $\mathrm{Or}$ il s'agit là de conditions que les atomes vérifient parfaitement. (L.R. 247 : 1136)

(13b) La solución ideal sería utilizar, según los mismos principios de un microscopio óptico o electrónico, partículas que tuvieran a la vez una débil energía cinética y una longitud de onda corta. Pero se trata de condiciones que los átomos cumplen perfectamente. (M.C. 130: 1010)

Le premier énoncé $\mathbf{p}$ présente de façon hypothétique une solution considérée comme idéale (des particules ayant simultanément une faible énergie cinétique et une courte 
longueur d'onde) et l'énoncé [or q] vient justement confirmer que cette solution existe réellement grâce aux caractéristiques des atomes. Une expression de confirmation s'impose donc dans ce cas:

[Precisamente se trata de condiciones que los átomos cumplen a la perfección.]

Cet emploi de $O R$, qui introduit un énoncé de type conclusif est, par son fonctionnement, à rapprocher de l'emploi signalé en (1a) "Or il n'en est rien», et en (2a) "Or cette hypothèse est impossible», que $\mathrm{j}$ 'ai appelé «réfutation» et qui lui est radicalement opposé.

\section{CONCLUSION}

Comme conclusion à cette analyse des emplois de $O R$, il me semble intéressant d'apporter quelques précisions sur la différence de fonctionnement entre ce connecteur français et le connecteur espagnol ahora bien que les dictionnaires bilingues proposent comme équivalent.

L'analyse du corpus montre en effet que dans la catégorie d'invalidation, $O R$ est traduit, dans la plupart des cas, par pero lorsqu'il exprime une opposition nette associée à une valeur de clôture, et par sin embargo ou no obstante lorsqu'il exprime une opposition plus nuancée. Quant à ahora bien, tout comme $O R$, il peut, théoriquement, être utilisé pour introduire aussi bien un argument anti-orienté qu'un argument non-orienté. Cependant, l'étude des textes révèle que ahora bien s'emploie pour exprimer une idée de poursuite et de développement de tout un mouvement argumentatif et que sa valeur argumentative est en quelque sorte éclipsée par sa valeur de marqueur de structuration. Il y a donc, à mon avis, une différence de fonctionnement entre ces deux marqueurs du point de vue du mouvement discursif qu'ils introduisent:

- $O R$ a la virtualité de convoquer de façon immédiate une valeur d'anti-orientation ou de co-orientation. Pour cette raison, on peut avoir une suite du type [p. $O R$ non-p], comme dans l'exemple (1) où $O R$ introduit une conclusion.

- Ahora bien, par contre, a une valeur argumentative "retardée» qui fait que l'argument qu'il introduit ne peut pas constituer en lui-même une conclusion mais seulement une étape vers une conclusion. Aussi, on trouvera rarement une forme du type:

[p. Ahora bien non-p],

[p. Ahora bien $\mathbf{q}]$.

mais plutôt

Por tanto,

soit non-p (invalidation)

soit $\mathbf{S}$ (argument non-orienté).

En résumé, on observe qu'il est donc extrêmement difficile pour les dictionnaires bilingues d'établir des correspondances «mot à mot», et plus particulièrement dans le cas des connecteurs, puisque l'interprétation de la valeur et de la fonction de ces «mots de liaison» ne repose pas seulement sur des relations au niveau de la microstructure, mais aussi sur un réseau de relations qui s'établissent au niveau macrotextuel. 


\section{NOTES}

1. Cette étude a été réalisée dans le cadre du projet de recherche «Análisis contrastivo de los elementos conectores de la argumentación y de los elementos temporales en textos de especialidad (francés/ castellano, inglés/castellano)» qui bénéficie d'une subvention du gouvernement espagnol DGICYT — Référence PB 95-0985-C03-01.

2. Les exemples sont extraits de la revue La recherche et de sa traduction en espagnol, Mundo Cientifico. La version espagnole est reproduite textuellement, étant donné que l'objectif de ce travail n'est pas de corriger la traduction, mais d'analyser un connecteur déterminé. Les références des exemples indiquent le numéro de la revue et la page.

3. La notion de «créateur d'univers» est empruntée à Adam (1990: 162).

4. J'emprunte la notion de mouvement discursif à Roulet (1985).

5. Les valeurs argumentatives de Ainsi ont été examinées dans Rey (1994).

\section{RÉFÉRENCES}

Adam, J. M. (1990): Éléments de linguistique textuelle, Liège, Mardaga. Anscombre, J. C. et O. Ducr ot (1983): L'argumentation dans la langue, Bruxelles, Mardaga. Del or S, J. (1992): Le nouveau concert européen, Paris, Éditions Odile Jacob.

Ducr ot, O. et al. (1980): Les mots du discours, Paris, Minuit.

Ducr ot, O. (1982): «Note sur l'argumentation et l'acte d'argumenter», Cahiers de linguistique française, 4, p. 143.

_ (1983) «Opérateurs argumentatifs et visée argumentative», Cahiers de linguistique française, 5, pp. 7-8.

Finger mann, G. (1972): Lógica y teoría del conocimiento, Buenos Aires, El Atheneo.

Lavaud, M. P. (1994): Pragmatique, logique naturelle et argumentation: le connecteur or et ses équivalents en espagnol, Thèse de doctorat, Université de Bourgogne.

M oeschl er, J. (1989a): La modélisation du dialogue, Paris, Hermès.

Raccah, P. Y. (dir.) (1992): L'argumentation dans le langage, Gand, Presses Universitaires.

Rey, J. (1994) : «La traducción del conector ainsi en textos especializados», Diálogos Hispánicos, 20, Amsterdam, Rodopi.

Roul et, E. (1985): L'articulation du discours en français contemporain, Berne, Peter Lang.

Spengl er , N. De (1980): «Première approche des marqueurs d'interactivité», Cahiers de linguistique française, 1, pp. 128-148.

Tricas, M. (1990): «L'argumentation concessive française et espagnole», Meta, 35 (3), pp. 529537.

(1995): Manual de traducción, Barcelona, Gedisa.

\section{Dictionnaires monolingues}

Le grand dictionnaire Larousse de la langue française (1989): Paris, Larousse.

Le Robert dictionnaire alphabétique et analogique de la langue française (1971): Paris, Le Robert. Le trésor de la langue française (1974): Paris, CNRS, Gallimard.

Diccionario de uso del Español (1984): de María Moliner, Madrid, Gredos.

Diccionario de la Lengua Española (1993): Madrid, Real Academia Española.

\section{Dictionnaire bilingues}

Le nouveau Larousse français-espagnol, espagnol-français (1989): de Ramón García Pelayo, Paris. Hispano-Bordas, dictionnaire français-espagnol, espagnol-français (1989): de Jean-Paul Vidal, Paris, Bordas. 